\title{
Blockchain and Internet of Things in Higher Education
}

\author{
Tanweer Alam*, Mohamed Benaida \\ Faculty of Computer and Information Systems, Islamic University of Madinah, Saudi Arabia
}

Received March 18, 2020; Revised April 12, 2020; Accepted April 27, 2020

Copyright $\odot 2020$ by authors, all rights reserved. Authors agree that this article remains permanently open access under the terms of the Creative Commons Attribution License 4.0 International License

\begin{abstract}
Building the innovative blockchain-based architecture across the Internet of Things (IoT) platform for the education system could be an enticing mechanism to boost communication efficiency among all participants within the $5 \mathrm{G}$ network. Wireless networking would have been the main research area allowing people to communicate without using the wires. It was established at the start of the Internet by retrieving the web pages to connect from one computer to another. Moreover, high-speed, intelligent, powerful networks with numerous contemporary technologies, such as low power consumption, and so on, appear to be available in today's world to connect among each other. The cloud features on physical things under IoT is allowed to store and process IoT and Blockchain data in any situation. One of the complex tasks throughout the area of mobile communications would be to design a new virtualization framework based on blockchain across the Internet of Things architecture. The goal of this research is to connect a new study for an educational system that contains Blockchain to the internet of things or keeping things cryptographically secure on the Internet. This research combines with its improved blockchain and IoT to create an efficient interaction system among students, teachers, employers, developers, facilitators, recruiters, and accreditors on the Internet. This specified framework is detailed research's great estimation.
\end{abstract}

Keywords Educational System, Internet of Things,
Wireless Communication, Blockchain, Cloud Computing

\section{Introduction}

For many decades, the education sector has experienced continuous technological advances. Currently, innovations such as Artificial Intelligence (AI), Virtual Reality (VR), Internet of Things, Blockchain, and wearable devices are being used to improve the education sector's ability. The proposed study is a move forward in the area of IoT in $5 \mathrm{G}$ diverse systems in which the authors propose a unique blockchain-based IoT structure of interacting different people and connected devices in the $5 \mathrm{G}$ network. That study result would be to introduce a new structure of communications on the IoT. The suggested study utilizes the required study's appropriate as well as effective simulation and could be introduced through an IoT structure. It seems that the whole universe is now becoming completely reliant on mobility facilities as well as wireless technology. The Blockchain (BC) throughout the IoT has become a novel innovation that behaves on a decentralized, distributed, public as well as a real-time database to collect operations among IoT endpoints [1]. Figure 1 demonstrates circumstances of the use of blockchain technology in 2019 throughout worldwide services according to the Statista report [2]. 


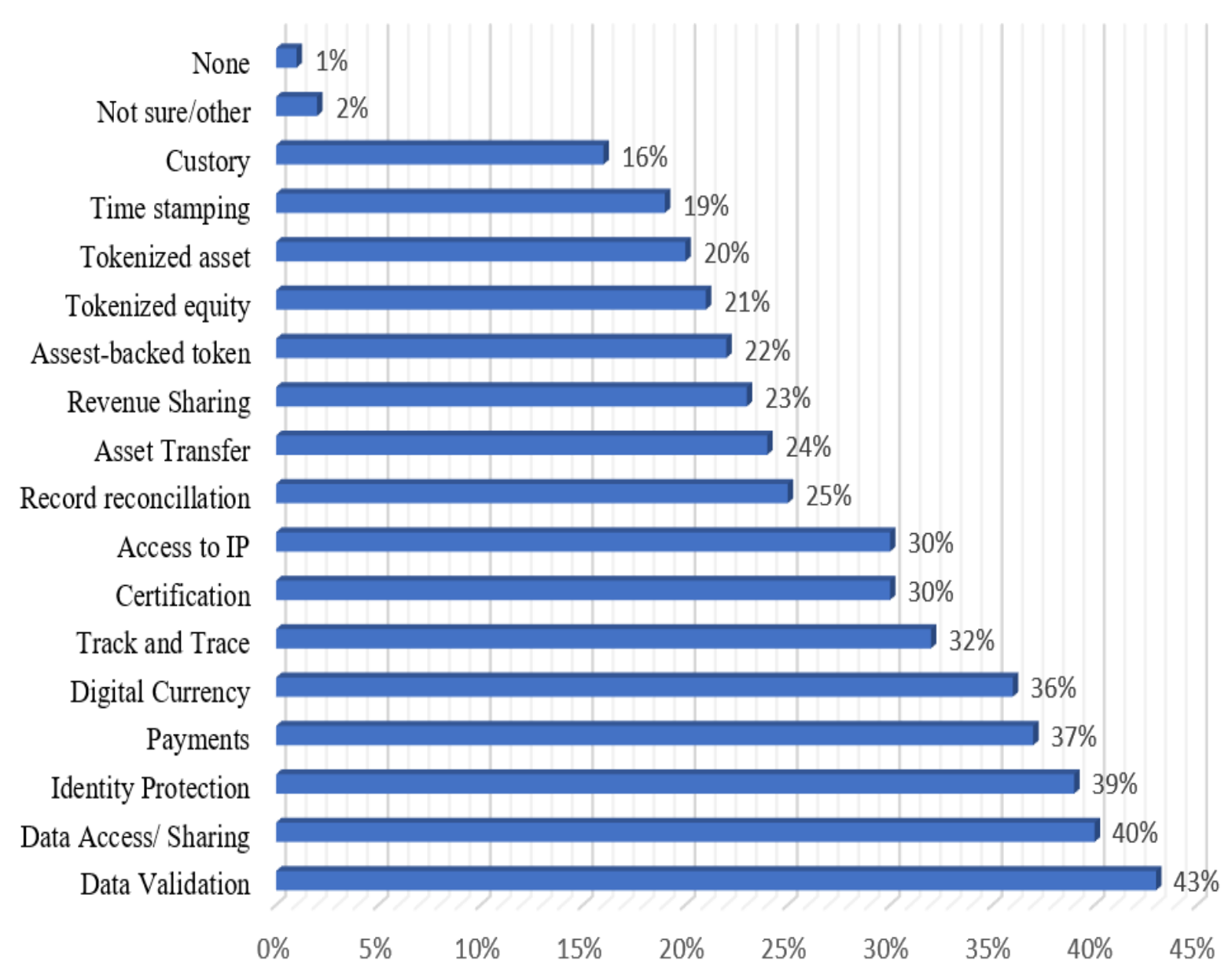

- Share of respondents

Figure 1. Statista Report on Blockchain technology use cases in organizations worldwide [2]

The blockchain is indeed a sequence of blocks where every block is connected to the prior blocks. Every block must have the cryptographically secure key, prior block hash, as well as its information. The BC operations will be the fundamental modules that had to transmit information among IoT endpoints. The IoT access points seem to be different kinds of natural however smart devices with integrated detectors, sensors, systems as well as worthy of interacting with several other IoT endpoints. BC's role in IoT would be to have a mechanism for handling protected information records by IoT endpoints [3]. BC seems to be a safe innovation that could be used openly as well as publicly. The Internet of things enables this technology to enable asymmetric cryptography among IoT endpoints in such a diverse system. BC transactions might be monitored as well as traversed through everything accessed to interact throughout the IoT. BC might well enhance interaction protection. The Internet of things has been increasing dramatically throughout the year with its objective in $5 \mathrm{G}$ innovations, like Smart Homes as well as smart Cities, e-education, e-Health, distributed intelligence, etc., but has privacy and security obstacles. The protection of confidentiality in connectivity among IoT gadgets paid too much publicity from 2017 to 2020 . The earlier studies committed to building or optimizing the connectivity structure; however, such a study may not produce its complete structure to IoT-BC communication among smart devices network [4].

This research strategy develops the study in and out of expanding the connectivity of things that utilize the fog as well as blockchain technology on networking [5]. Exchange information from one specification to the next using wireless communication begins in the form of the radio network packets starting from the 1973 year. A Machine was able to connect with another machine of the same specification. There is a huge number of articles published by several other authors on blockchain as well as the internet of things in between 2017-2020. Figure 2 represents the framework where the teachers, students, operators, employers, and accreditation unit members can communicate using the blockchain-IoT framework. 


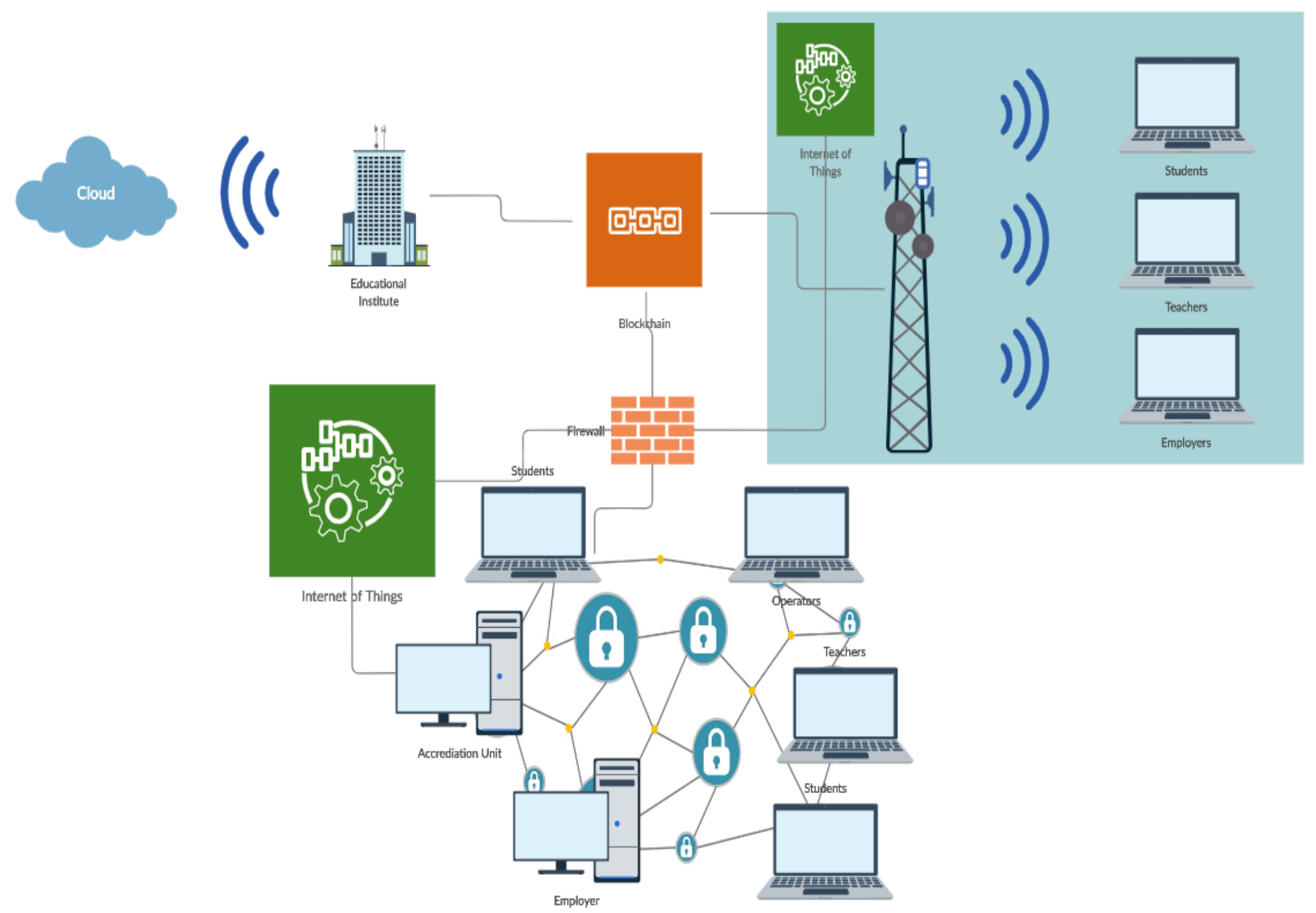

Figure 2. Blockchain-IoT framework for the educational system 
In such a decentralized strategy, the IoT users have been directly linked. It is therefore much more complicated to use the conventional current security strategies in the interaction among IoT users. $\mathrm{BC}$ is an innovation that provides security in transactions among IoT users [6]. This offers a decentralized, distributed as well as publicly available mutual ledger to collect blocks information which is stored or confirmed in such an IoT system. Its information stored throughout the distributed ledger is immediately attempted to use peer-to-peer configuration. The $\mathrm{BC}$ is an innovation at which IoT endpoints handle the transactions in the type of such a block in the blockchain [7]. The following requirements are needed to have a blockchain in higher education.

\subsection{Identification and Academic Reports}

Universities of education face difficulties in preserving the learners' identification, essential expertise or information.

\subsection{Modern Pedagogy}

Every learner does have its learning skills. It is important to build new educational frameworks to meet each student's needs.

\subsection{Student Loans}

Spending on education convinces learners to choose a profession of preference. Learner's incentive services have been designed to recognize the talents of the learners.

\subsection{Higher Education}

Implementing new higher education frameworks to fulfill expected desires or making the dream come true.

The objective of this proposed strategy is to bring the new idea where the stockholders in the education sector incorporate together as well as join a group and facilitate secure communication among them. This really improves the IoT security and offers secure or authentic interaction among all the users. The objective of this study is to develop a communication structure for the educational system and to provide a trustworthy, safe and quick link among connected users on the Internet of Things using blockchain technology.

The organization of the rest of the research paper is as follows: Section 1-Introduction presents a brief overview of this research paper. Section 2 - why blockchains?, Section 3 - the Blockchain and IoT in Higher Education and Section 4 - describes the Opportunities for the Proposed Approach in the Educational System, section 5 shows Challenges, Section 6 shows the applications and section 7 represents the conclusion of the research paper and the future scope of the proposed study.

\section{Why Blockchain?}

Packets are transmitted in cloud using a Blockchain node. Each node is also called a block. Every block has its hash code (unique id like a fingerprint), previous block hash code and their data. Every node has a connection to exchange information. Every connection to exchange information contains a lot of blocks with its previous blocks hash code and data. These blocks are connected with security through cryptography techniques. The blockchain nodes are like the linked list node in the data structure. So, we can say that blockchain is a complex data structure. The blocks are distributing in a decentralized system using the point-to-point topological network [8], [9]. When a new block is created, then it moves to the network and visits every connected node and checks its authentication. If it is valid, then it will connect to the blockchain and its hash will generate only once. This newly generated block stores the hash of the previous block and connects to the chain. The performance of the proposed system is evaluated through different experiments. Firstly, the authors have created thousands of blocks with a fixed size by using the open-source software (Node.js). The authors have created the IoT network, connected with cloud and, created fog and blockchain. This suggested structure depicted the use of Fog computing with IoT gadgets onto the edge of the network using blockchain technology to communicate, exchange and share information amongst the IoT endpoints in the educational sector. Transactions in the suggested structure were transferred in the point-to-point network topology. There are some unique IoT endpoints termed as Miners throughout the system. These are usually used to verify network transactions. According to Whether the transactions are confirmed or not, they are transformed into the blocks or decided to add to the existing Blockchain or transmitted to the channel. Its miners play a vital role in adapting the creation of a new block in the blockchain. Throughout this study, we used many examinations to determine the structure. A hashing algorithm is introduced using the IROHA tool of Hyper ledger. A Docker-compose is built on the device. A Hyperledger IROHA tool involves several services like decentralized Hyperledger, Proof of Work (PoW) algorithms, P2P system, and so on. Sumeragi in Hyperledger IROHA methodology is introduced in Blockchain. An IROHA Android and iOS packages need the facility with the blockchain to communicate with the IoT endpoints. According to the Sumeragi algorithm, IoT nodes have requested the transactions or followed the basic steps:

Step 1. Transmitting:

The members confirm, arrange or sign the transactions or send data to the system [10].

Step 2. Authentication as well as going to sign:

This validates, requests or signs the transaction or broadcasts to the peer-to-peer channel's approved IoT 
endpoint.

Step 3. Committed:

Dedicate regarding signing.

Throughout the incident of server's failure, the automated system contributes a move named error detection. Also, the algorithm works with the existing server to monitor the inconsistencies.

Consider the newly created block node that is referenced by two previously created blocks. The node is placed between these two blocks. Consider that the block is verified by the miners, the time representes the verification time calculated by the Poisson process [11]. When a new block is created, then it moves to the network and visits every connected node and checks its authentication. If it is valid, then it will connect to the blockchain and its hash will generate only once. This newly generated block stores the hash of the previous block and connects to the chain.

Blockchains should impact on the higher education system such as business schools that are more urgent or far-reaching than the other widely mentioned innovations like artificial intelligence and robotics. Although these innovations provide significant learning and convening ability, the blockchains could significantly, by changing the way the school of business, conducts its practice.

This study is proposed to build up an educational system depending on blockchain and IoT. Building a trusted educational credit and grading framework could be of benefit to the blockchain technology. Proof of concept is introduced using Ark blockchain open-source framework. This platform suggested addresses learners and institutions from a worldwide homogeneous viewpoint. Learners benefit from either a single or transparent perspective of their finished classes, whereas the model has access to state-of-the-art information irrespective of the academic background of a learner. Many recipients of the proposed change are prospective employers, who are able to immediately verify the student's information. This solution implemented is focused on decentralized peer-to-peer network mechanism. This really exchanges the higher education grading system to an effective, streamlined, pervasive model based on blockchain from current global-world physiological documents or conventional electronic documents. This is a process to ultimately adapt into a homogeneous, streamlined, and worldwide pervasive credit and grading system for higher education.

\section{Blockchain and IoT in Higher Education}

Blockchains are beneficial if data needs to be shared by various parties without trust. A blockchain is a means of sending data in a completely automated and secure manner, as falsification is virtually impossible. A blockchain is a time-stamped sequence of an unchanging information record that is attempted by a cluster of computer systems not operated by any single user. Not conventional database blockchains are distributed, and everybody has to accept to make any changes to manage it. The biggest achievement behind this study has been building a structure for the educational system to communicate using blockchain technology and IoT on the Internet [12]. This framework becomes primarily suitable for the users where information becomes regularly conveyed to the connected devices on the internet network. In addition, we used a retransmission strategy, variable packet length, or populated congestion situation to improve the proposed system. A plan of action for this study has been defined. For performance onto the Internet of things, the execution of the IoT-BC structure to interact safely between IoT devices will be programmed [13]. The whole study would be implemented as a three-layer structure, such layers becoming Fog, Blockchain, and IoT layers. This research supports wireless communication to develop an IoT-Blockchain framework between several users on the IoT. An IoT-Blockchain framework does have the following elements:
a) Smart Devices
b) Network
c) Development tools
d) Blockchain

Figure 3 shows the blocks in a blockchain.

Blockchain Technology (BT) in IoT provides a mechanism of processing protected information files by IoT endpoints. BT transactions might be monitored or examined through someone verified to interact throughout the IoT system. An IoT-BC could enhance interaction security.

BC's major advantages with IoT are:

1) Building trust between IoT public endpoints or minifying the possibility of accidents.

2) Minifying transportation costs by communicating directly without third parties.

3) Speeding up transactions in real-time.

The visualization servers contained efficient services in the fog, the intermediaries are 3rd-party servers that could also store secured information. A token offered by the approved Blockchain repository to the smart device does have the power to connect the model, notification key from the use of a key computer, collect information from the fog [14]. 


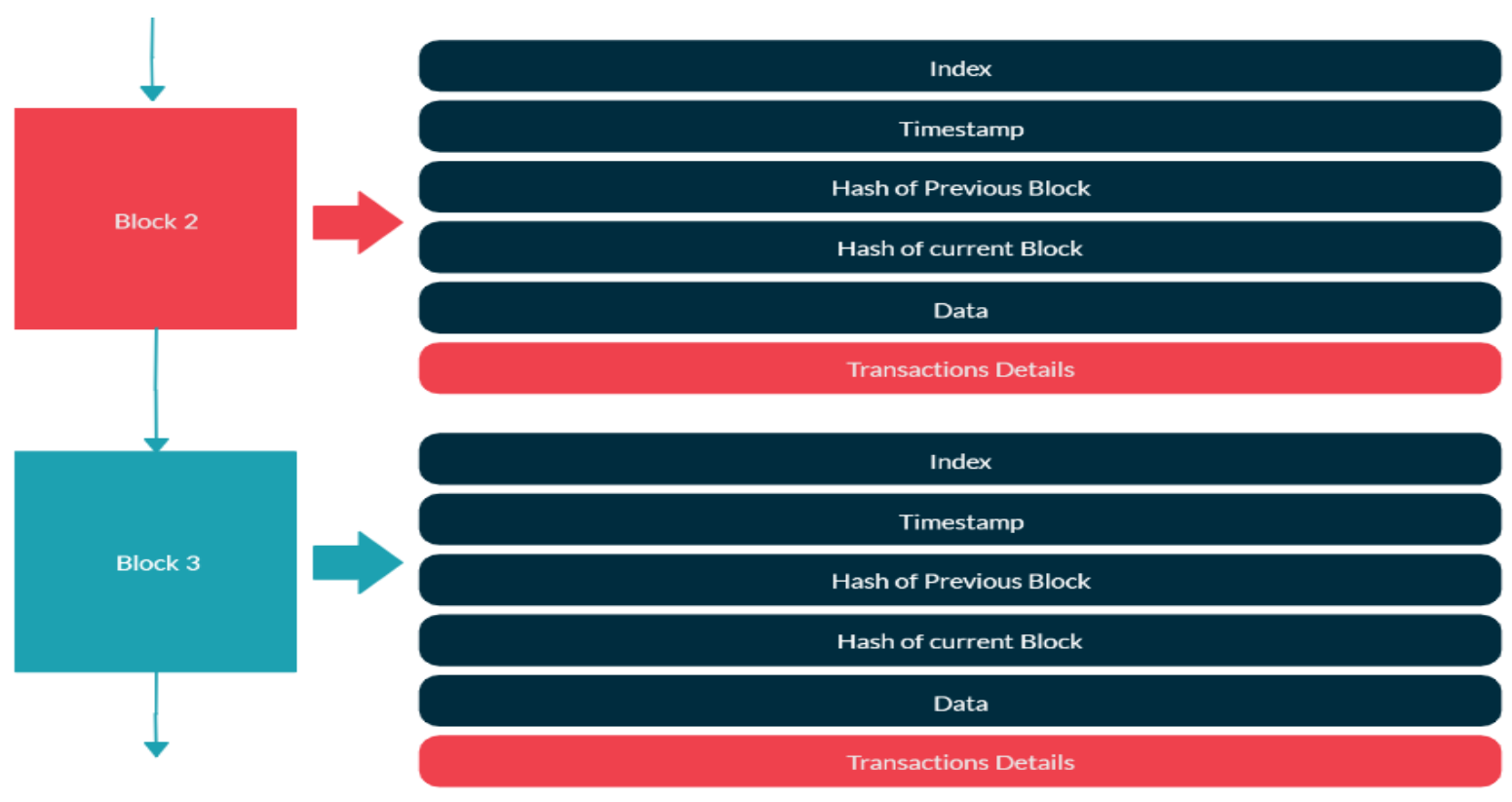

Figure 3. Blockchain's Blocks

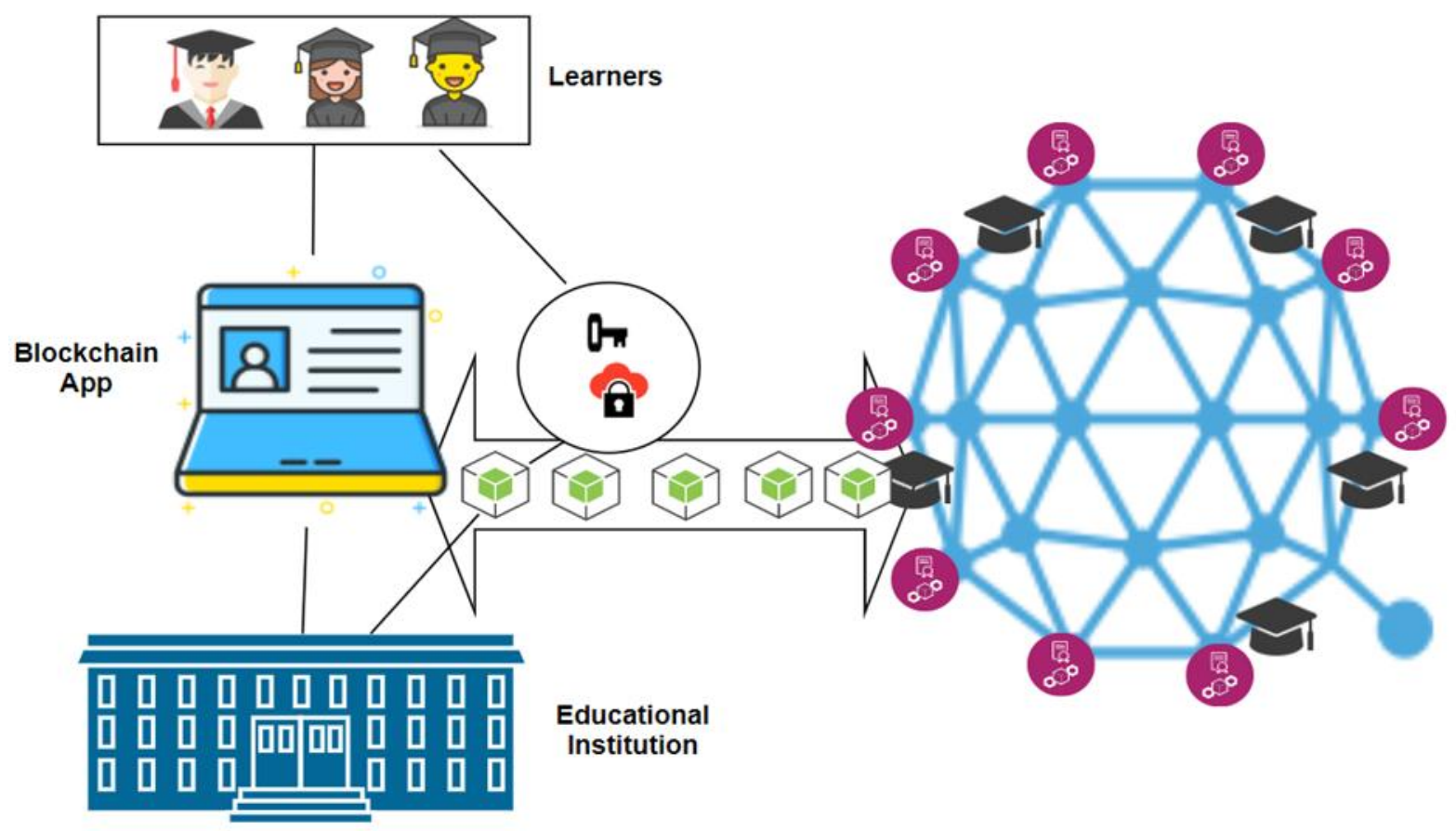

Figure 4. Blockchain-based Educational System

Several smart contracts released in the approved Blockchain repository through the visualization servers, intermediaries or fog proprietors. IoT's portable device discovers the smart contacts from within the permitted Blockchain repository [15]. Its permitted Blockchain repository produces the token to IoT's smart device. A smart device requests the keys in middleware from a key server as well as directs the token with the query. A primary server verifies the token from such an approved blockchain repository or produces a key for each smart device as well as a reaction down to a device [16]. Once again, the IoT smart device is permitted to retrieve information from the cloud. The IoT nodes might be a student, a teacher, an employee, an operator or an accreditation unit member. They all have smart devices for communication within the system (Figure 4).

A blockchain with IoT functions together with its goals that could be summarized.

1) Decentralization structure

Internet of things as well as BC, both approaches would be identical. This structure eliminates the centralized 
approach or even provides the facility for a decentralized architecture. This enhances the aggregate control system probability of failure or efficiency.

\section{2) Protection}

Transactions among endpoints users were often protected throughout the $\mathrm{BC}$. This is a different approach to secure interactions. The $\mathrm{BC}$ enables IoT users to interact reliably with one another.

\section{3) Identifier}

Both connected devices have always been distinctively recognized with such a cryptographic signature in IoT. Every block of BC is often distinctively recognized. Therefore, $\mathrm{BC}$ is a trustworthy innovation that offers distinctively recognized information which is accessed throughout a distributed ledger.

\section{4) Accuracy}

Internet of things endpoints in $\mathrm{BC}$ has become capable of accessing the information being passed on the Internet. Its information has been accurate when it is confirmed by the miners once joining BC. Just confirmed blocks could indeed join the BC.

\section{5) Independent}

All IoT endpoint users have become available to interact with any computer cluster with a decentralized framework.

\section{6) Optimization}

IoT users could interact in high-availability, a decentralized intelligent network that communicates to the destination device in real-time or transaction data.

The Internet of things enables the linked physical objects to exchange their data throughout the diverse system. It could be separated into the following points.

\section{1) Smart Devices}

The Internet of things assigns the distinctive identification number to each connected device in the system. These devices could exchange information among the IoT endpoints.

\section{2) Routers}

The routers seem to be the machines that operate among physical objects as well as the cloud to make sure that now the link is maintained and therefore that protection has been granted to the network system [17].

\section{3) Building a network}

It would be used to manage the flow of data and to maintain the quickest route amongst the other IoT endpoints.

\section{4) Cloud}

It would be used to deposit or determine information.

A repository in Blockchain is said to have characteristics including a distributed security model, restricted access, higher public access, bottom to top confidentiality or exchangeable identifications, whilst in a central database, the characteristics have become a centralized trust model, low security, low public access, high confidentiality but also un-transferable identifications [18]. A blockchain is now more sophisticated than that of the centrally controlled storage in the above characteristics.

\section{Opportunities for the Proposed Approach in the Educational System}

The smart classrooms are not too far away when they will integrate with blockchain and Internet of Things technologies. These technologies will become an extremely important part of academies around the world shortly. The question is, How's the system going to benefit the employers as well as academics? the answer is yes, realizing the full influence of blockchain on the education system or determine that this innovation would have an impact on teacher and student, this is useful to know how some other sectors have used this to enhance the mechanisms and also how universities could one day follow their lead.

A BC-IoT interoperability strategy does have many extraordinary possibilities in the educational system. This unlocks the two new windows next to each other. Several of the possibilities would be characterized as follows.

\subsection{Constructing the Trust among Parties}

Due to its highly advanced features, the BC-IoT strategy would develop a relationship amongst the multiple smart devices. Just confirmed gadgets could interact in the system, as well as the miners would first confirm every block of the transaction, then they should join the BC [19].

\subsection{Lower Costs}

Such a strategy would lower costs as it interacts effectively without the third party. This removes all third-party nodes among the recipient as well as the sender. This strategy offers full correspondence [20], [21].

\subsection{Minimize Time}

It reduces a huge amount of time. This strategy minimizes the transaction time from weeks to seconds. For example, in the traditional approach, when students want to take admission in the university, he pays a lot of time to fill forms and wait for the reply from the university 
administration.

\subsection{Security and Privacy}

It offers privacy and security to users, devices, and data.

\subsection{Public Services}

This approach offers services to the public sector. Authenticated users could connect and exchange information [22].

\subsection{Investment Management}

Such an approach safely exchanges money without the need for a third party. This offers quick, safe as well as financial information facilities. This lowered the cost or time of transition [23].

\subsection{Risk Management}

Such an approach plays a vital role in examining or reducing the chance of resource or transaction failure.

\subsection{Educational Institution Activities}

Blockchains also can change certain roles at educational institutions. The blockchain would become a great format for granting qualifications and experience, that would remove the institution administrator's role as the site of delivery. This would also reduce a stream of institution profits, this might reduce the costs or allow those services to be distributed anywhere.

Currently higher education institutions around the globe are introducing their people to blockchain courses as they recognize the value under the technological advances. Many of the nations which have blockchain educational institutions united states, Canada, Denmark, Singapore, Spain, etc. The Cornell University, in the US, is one of the first universities to introduce blockchain-focused courses into their syllabus.

\section{Challenges}

The proposed framework might experience several difficulties like scaling, storing, skills and discovery, and so on. The following is also the challenge facing the interoperability strategy.

\subsection{Usability}

The BC could hang due to its high transaction load. In 2019, the Cryptocurrency warehousing has become more than 197 GB capacity [24]. Assume when IoT is incorporated into $\mathrm{BC}$ so the load would be heavier than that of the present situation.

\subsection{Storing Capacity}

An electronic ledger would be preserved by each IoT endpoint. Throughout time, this will improve its storage size, that will be a difficult task or become a huge load on every other wireless device.

\subsection{Abilities Absence}

$\mathrm{BC}$ is now a new revolutionary technology. Few people around the world understand this technology.

\subsection{Finding or Convergence}

$\mathrm{BC}$ may not be originally designed for IoT. Finding a further gadget in BC or IoT is a really difficult task for all the smart devices. Internet of things endpoints could find one another; however, they likely won't find or incorporate the $\mathrm{BC}$ with yet another gadget.

\subsection{Confidentiality}

Its ledger would be dispersed openly to every linked network. Every connected device could look at the transactions of the blockchain. Confidentiality has always been a difficult task in the embedded strategy.

\subsection{Compatibility}

The BC could be whether public or private. Therefore, scalability around public and private Blockchain would also be a difficulty in the BC-IoT strategy.

\subsection{Laws and Regulations}

An IoT-BC is acting worldwide, and therefore it faces several regulations to implement this strategy worldwide.

\section{Applications}

In higher education, blockchain has several applications, but this technology has not entered yet the public consciousness. It's just that, there is no lack of startup companies and major players intending to make that possible. But here are some instances of blockchain often used in the educational system around the world. Learning is becoming the essential part of our life. This is the base for innovations, too. The Blockchain and IoT are the aspect of such innovation which the educational institutions can profit greatly from. Most of the educational resources are available with the advent of technology in smart devices. The following are some Blockchain applications used for educational purpose.

\subsection{Blockcerts}

A system for developing, granting and confirming 
educational credentials [25]. It is an open platform for developing applications that validate or authenticate blockchain-based government documents. This may involve certificates for municipal data, academic qualifications, skilled licenses, the growth of the labor force and so on. It includes a number of open-source collections, techniques and mobile applications that enable a decentralized, standard-based, beneficiary-centric environment that enables untrustworthy verification through blockchain ledger. The users of Blockcerts utilizes and motivates the consolidation of open platforms. It is dedicated to the auto-sovereign identity for all individuals and enables recipients to monitor their claims via easier-to-use methods like the Mobile Applications. Figure 5 represents the working process of Blockcerts application [25].

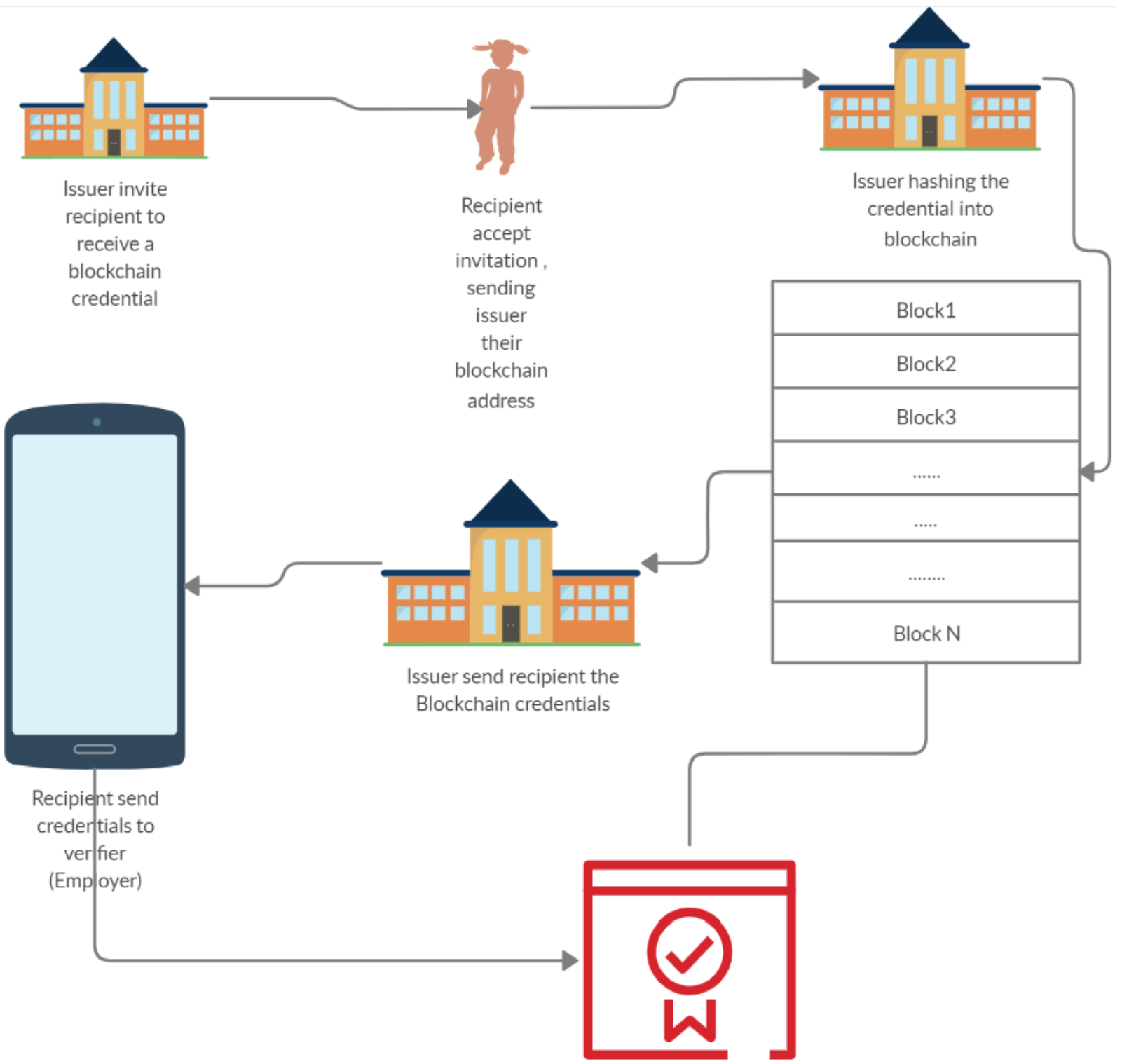

Verifier checks the

blockchain to verify

the certificate

Figure 5. Working process of Blockcerts Application 


\subsection{APII}

Confirm the academic qualifications of potential students and teachers [26]. This application expects to maximize everybody's ability and has functionalities on its website and mobile applications that are doing this. Individual people can maximize their capability and enterprises can minimize their charges and administration while quickly finding learners and skills. Find easy direct connections to your web pages, fingerprint verification, professional verification records and a nano-accreditation generate mechanism utilizing QR coding [26].

\subsection{ODEM}

A platform that links teachers, students, and professionals to relevant courses and services [27]. This is a blockchain-powered framework in which learners can improve their educational progress effectively with academics and the potential employers, and they also often establish useful, energetic syllabuses. By using smart contracts to automatic payment, ODEM is easy to handle and much more cost effective to learn. Entering thousands of ODEM students from all over the world, it gets adequately compensated low-cost, student-driven market, allowing them to focus on what they do strongest: motivating curious students who enjoy learning [27].

\subsection{Sony Global Education}

A website that maintains a consistent record of students' information and educational accomplishments [28]. It introduces a new transcript and score management service via a digital service called Blockchain technology. It demonstrates how well the future of establishing and handling transcripts and high-security information in education will become a blockchain mechanism. This is also demonstrated by the use of blockchain for the installation of the "Next Generation of School ICT Environment" for the Japan's Ministry of Internal Affairs and Communications [28].

\subsection{Parchment}

It is a forum for assessing academic qualifications, processing programs and generating certificates. Learners can discuss their academic performance with prospective employers with ease [29]. It is the most broadly accepted electronic credential service that allows learners, academic institutions and employers to apply for, verify and share their credential in an easy and secure environment. Its framework has helped millions of people and thousands of colleges and universities transfer more than 30 million transcripts and other certificates worldwide [29].

\section{Conclusions}

This study links the blockchain and IoT for the educational system, where IoT and blockchain technologies are utilizing. The proposed research shows a combination of the IoT and blockchain for the educational system. The Blockchain is used to create a hyper-distributed public authentic ledger to record the transactions. The research opened a new opportunity in this area. The framework is implemented using a different set of IoT nodes and tested. This study can be a valuable framework to improve the communication security and efficiency in the educational system. This framework is appropriate for providing communication security where huge data is transmitted in a heterogeneous environment in the future. We have tested the system in different scenarios such as memory and processor usage in the integrated system and its impact on the performance of overall the system. We found that the proposed framework, not only increases the throughput but also the direct connection among IoT nodes are established, which makes the system more stable. The outcomes of this research established a new IoT framework with blockchain technology for the existing educational system. In the future, researchers can enhance this research and apply it for whole the kingdom.

\section{REFERENCES}

[1] Yi, Shanhe, Zijiang Hao, Zhengrui Qin, and Qun Li, Fog computing: Platform and applications, In 2015 Third IEEE Workshop on Hot Topics in Web Systems and Technologies (HotWeb), pp. 73-78, IEEE, 2015.

[2] Statista, URL: https://www.statista.com/statistics/878732/w orldwide-use-cases-blockchain-technology/

[3] Tanweer Alam. "Internet of Things: A Secure Cloud-Based MANET Mobility Model." International Journal of Network Security, Vol. 22(3), 2020.

[4] Tanweer Alam. "Efficient and Secure Data Transmission Approach in Cloud-MANET-IoT integrated Framework.", Journal of Telecommunication, Electronic and Computer Engineering (JTEC). Vol. 12 No. 1, 2020.

[5] Tanweer Alam, Mohamed Benaida. "The Role of Cloud-MANET Framework in the Internet of Things (IoT).", International Journal of Online Engineering (iJOE), Vol. 14(12), pp. 97-111, 2018.

[6] Tanweer Alam. "Middleware Implementation in Cloud-MANET Mobility Model for Internet of Smart Devices." International Journal of Computer Science and Network Security, 17(5), pp. 86-94, 2017.

[7] Tanweer Alam, Mohamed Benaida. "CICS: Cloud-Internet Communication Security Framework for the Internet of Smart Devices.", International Journal of Interactive Mobile Technologies (iJIM), vol12(6), pp.74-84, 2018.

[8] Mohammed Aljohani, and Tanweer Alam. "An algorithm for accessing traffic database using wireless technologies.", International Conference on Computational Intelligence and Computing Research (ICCIC), pp. 1-4, IEEE, 2015. 
[9] Tanweer Alam, and Mohammed Aljohani. "Design a new middleware for communication in ad hoc network of android smart devices." Second International Conference on Information and Communication Technology for Competitive Strategies, p. 38. ACM, 2016.

[10] Tanweer Alam. "Fuzzy control based mobility framework for evaluating mobility models in MANET of smart devices.", ARPN Journal of Engineering and Applied Sciences, vol 12, no. 15, pp. 4526-4538, 2017.

[11] Tanweer Alam, Mohamed Benaida. "Blockchain, Fog and IoT Integrated Framework: Review, Architecture and Evaluation.", Technology Reports of Kansai University, Volume - 62, Issue 02, 2020.

[12] Sergio, FT de O., Joao F. da Silva Junior, and Fernanda MR de Alencar, "The Blockchain-based Internet of Things Development: Initiatives and Challenges", ICSEA, Vol 39, 2017.

[13] Dorri, Ali, Salil S. Kanhere, and Raja Jurdak, "Blockchain in internet of things: challenges and solutions", arXiv preprint arXiv:1608.05187, 2016.

[14] Samaniego, Mayra, and Ralph Deters, "Blockchain as a Service for IoT" In Internet of Things (iThings) and IEEE Green Computing and Communications (GreenCom) and IEEE Cyber, Physical and Social Computing (CPSCom) and IEEE Smart Data (SmartData), 2016 IEEE International Conference on, 433-436, IEEE, 2016.

[15] Kuzmin, Alexander, "Blockchain-based structures for a secure and operate IoT", Internet of Things Business Models, Users, and Networks, IEEE, 2017.

[16] Han, Runchao, Vincent Gramoli, and Xiwei Xu, "Evaluating Blockchains for IoT", New Technologies, Mobility and Security (NTMS), 9th IFIP International Conference on, IEEE, 2018.

[17] Song, Juah C., et al.,"Blockchain Design for Trusted Decentralized IoT Networks", 13th Annual Conference on System of Systems Engineering (SoSE), IEEE, 2018.

[18] Panarello, Alfonso, Nachiket Tapas, Giovanni Merlino, Francesco Longo, and Antonio Puliafito, "Blockchain and IoT Integration: A Systematic Survey", Sensors, vol 8, no. 8, 2018.

[19] Turkanović, Muhamed, Marko Hölbl, Kristjan Košič, Marjan Heričko, and Aida Kamišalić, "EduCTX: A blockchain-based higher education credit platform", IEEE access, 2018.

[20] Tanweer Alam. "IoT-Fog: A Communication Framework using Blockchain in the Internet of Things.”, International Journal of Recent Technology and Engineering (IJRTE), Volume-7, Issue-6, 2019.

[21] Tanweer Alam. "A reliable framework for communication in internet of smart devices using IEEE 802.15.4.", ARPN Journal of Engineering and Applied Sciences, Vol 13(10), 3378-3387, 2018.

[22] Tanweer Alam, and Mohammed Aljohani. "Design and implementation of an Ad Hoc Network among Android smart devices.", International Conference on Green Computing and Internet of Things (ICGCIoT), pp. 1322-1327, IEEE, 2015.
[23] Tanweer Alam, and Mohammed Aljohani. "An approach to secure communication in mobile ad-hoc networks of Android devices." International Conference on Intelligent Informatics and Biomedical Sciences (ICIIBMS), pp. 371-375. IEEE, 2015.

[24] Marsalek, Alexander, Thomas Zefferer, Edona Fasllija, and Dominik Ziegler. "Tackling data inefficiency: Compressing the bitcoin blockchain." In 2019 18th IEEE International Conference on Trust, Security and Privacy In Computing And Communications/13th IEEE International Conference On Big Data Science And Engineering (TrustCom/BigDataSE), pp. 626-633. IEEE, 2019.

[25] Blockcerts Universal Verifier. URL: https://www.blockcert s.org/

[26] Employee background checks and CV verification underpinned by blockchain technology. URL: https://appii. io/

[27] The ODEM Trust Network. URL: https://odem.io/odem-tru st-network/

[28] Sony Global Education URL: https://www.sonyged.com/

[29] Turn Credentials into Opportunities. URL: https://www.par chment.com/ 